\title{
Gut Microbiota Interplay With COVID-19 Reveals Links to Host Lipid Metabolism Among Middle Eastern Populations
}

\begin{abstract}
Mohammad Tahseen Al Bataineh 1,2,3,4t, Andreas Henschel/,5t, Mira Mousa ${ }^{3,6}$, Marianne Daou', Fathimathuz Waasia ${ }^{3}$, Hussein Kannout ${ }^{3}$, Mariam Khalili ${ }^{3}$, Mohd Azzam Kayasseh ${ }^{8}$, Abdulmajeed Alkhajeh ${ }^{9}$, Maimunah Uddin ${ }^{10}$, Nawal Alkaabi ${ }^{10}$, Guan K. Tay 11,12, Samuel F. Feng ${ }^{3,13}$, Ahmed F. Yousef ${ }^{7}$ and Habiba S. Alsafar ${ }^{3,13,14 *}$ on behalf of the UAE COVID-19 Collaborative Partnership
\end{abstract}

\section{OPEN ACCESS}

Edited by:

Davide Zella,

University of Maryland, Baltimore,

United States

Reviewed by:

Sebastian Schloer,

University of Münster, Germany

Ravi Kant Narayan,

All India Institute of Medical Sciences

(Patna), India

Yixin Xie,

The University of Texas at El Paso,

United States

*Correspondence:

Habiba S. Alsafar

habiba.alsafar@ku.ac.ae

†These authors share first authorship

Specialty section:

This article was submitted to Infectious Agents and Disease,

a section of the journal

Frontiers in Microbiology

Received: 19 August 2021 Accepted: 30 September 2021

Published: 05 November 2021

Citation:

Al Bataineh MT, Henschel A,

Mousa M, Daou M, Waasia F Kannout $H$, Khalili M, Kayasseh MA, Alkhajeh A, Uddin M, Alkaabi N, Tay GK, Feng SF, Yousef AF and Alsafar HS (2021) Gut Microbiota Interplay With COVID-19 Reveals

Links to Host Lipid Metabolism Among Middle Eastern Populations.

Front. Microbiol. 12:761067.

doi: 10.3389/fmicb.2021.761067
'Sharjah Institute for Medical Research, University of Sharjah, Sharjah, United Arab Emirates, ${ }^{2}$ Department of Clinical Sciences, College of Medicine, University of Sharjah, Sharjah, United Arab Emirates, ${ }^{3}$ Center for Biotechnology, Khalifa University of Science and Technology, Abu Dhabi, United Arab Emirates, ${ }^{4}$ Department of Genetics and Molecular Biology, Khalifa University of Science and Technology, Abu Dhabi, United Arab Emirates, ${ }^{5}$ Department of Electrical Engineering and Computer Science, Khalifa University, Abu Dhabi, United Arab Emirates, ${ }^{6}$ Nuffield Department of Women's and Reproduction Health, Oxford University, Oxford, United Kingdom, ${ }^{7}$ Department of Chemistry, Khalifa University of Science and Technology, Abu Dhabi, United Arab Emirates, ${ }^{8}$ Emirates Specialty Hospital, Dubai Healthcare City, Dubai, United Arab Emirates, ${ }^{9}$ Medical Education and Research Department, Dubai Health Authority, Dubai, United Arab Emirates, ${ }^{10}$ Department of Pediatric Infectious Disease, Sheikh Khalifa Medical City, Abu Dhabi, United Arab Emirates, ${ }^{11}$ Division of Psychiatry, Faculty of Health and Medical Sciences, The University of Western Australia, Crawley, WA, Australia, ${ }^{12}$ School of Medical and Health Sciences, Edith Cowan University, Joondalup, WA, Australia, ${ }^{13}$ Department of Mathematics, Khalifa University of Science and Technology, Abu Dhabi, United Arab Emirates, ${ }^{14}$ Department of Biomedical Engineering, Khalifa University of Science and Technology, Abu Dhabi, United Arab Emirates

The interplay between the compositional changes in the gastrointestinal microbiome, severe acute respiratory syndrome coronavirus 2 (SARS-CoV-2) susceptibility and severity, and host functions is complex and yet to be fully understood. This study performed 16S rRNA gene-based microbial profiling of 143 subjects. We observed structural and compositional alterations in the gut microbiota of the SARS-CoV-2infected group in comparison to non-infected controls. The gut microbiota composition of the SARS-CoV-2-infected individuals showed an increase in anti-inflammatory bacteria such as Faecalibacterium $\left(p\right.$-value $\left.=1.72 \times 10^{-6}\right)$ and Bacteroides $(p$ value $=5.67 \times 10^{-8}$ ). We also revealed a higher relative abundance of the highly beneficial butyrate producers such as Anaerostipes $\left(p\right.$-value $\left.=1.75 \times 10^{-230}\right)$, Lachnospiraceae $\left(p\right.$-value $\left.=7.14 \times 10^{-65}\right)$, and Blautia $\left(p\right.$-value $\left.=9.22 \times 10^{-18}\right)$ in the SARS-CoV-2-infected group in comparison to the control group. Moreover, phylogenetic investigation of communities by reconstructing unobserved state (PICRUSt) functional prediction analysis of the 16S rRNA gene abundance data showed substantial differences in the enrichment of metabolic pathways such as lipid, amino acid, carbohydrate, and xenobiotic metabolism, in comparison between both groups. We discovered an enrichment of linoleic acid, ether lipid, glycerolipid, and glycerophospholipid metabolism in the SARS-CoV-2-infected group, suggesting a link to SARS-CoV-2 entry and replication in host cells. We estimate the major contributing genera to the four pathways to be Parabacteroides, Streptococcus, 
Dorea, and Blautia, respectively. The identified differences provide a new insight to enrich our understanding of SARS-CoV-2-related changes in gut microbiota, their metabolic capabilities, and potential screening biomarkers linked to COVID-19 disease severity.

Keywords: COVID-19, glycerophospholipid, linoleic acid, microbiota, SARS-CoV-2

\section{INTRODUCTION}

The newly emerged $\beta$-coronavirus, severe acute respiratory syndrome coronavirus 2 (SARS-CoV-2), was identified as the cause of the respiratory illness coronavirus disease-2019 or COVID-19. It was first reported in Wuhan, China, in late 2019, after which it spread rapidly worldwide, with an alarmingly high transmission rate. Aside from the commonly reported respiratory symptoms, including fever, chills, and shortness of breath, more than $20 \%$ of patients have also been shown to suffer from gastrointestinal symptoms, such as diarrhea, nausea, abdominal pain, and vomiting (He et al., 2020).

Severe acute respiratory syndrome coronavirus 2 invades human cells through the interaction of its surface spike protein with angiotensin-converting enzyme 2 (ACE2) receptors expressed on the surface of several human cell types ( $\mathrm{Li}$ et al., 2020). ACE2 is predominantly expressed in human lung tissue, which correlates with the primary COVID-19 infection site (Wölfel et al., 2020). However, ACE2 receptors are also highly expressed on enterocytes and colonocytes that line the intestinal epithelium (Lee et al., 2020; Villapol, 2020). Together, the presence of ACE2 receptors in gut epithelia and the gastrointestinal symptoms of COVID-19-infected individuals suggest that the gastrointestinal tract is an extrapulmonary site for SARS-CoV-2 activity and infection (Hoffmann et al., 2020; Zuo et al., 2020b).

The gastrointestinal symptoms have been linked to the dysbiosis of the intestinal microbiome, resulting from the viral infection and the subsequent alterations of the immune response ( $\mathrm{He}$ et al., 2020). Invading viruses can alter host immune responses by facilitating stimulatory or suppressive responses usually regulated by the microbiota in the gut (Zuo et al., 2020b). Moreover, viral infections can alter the gut microbiome composition resulting in the depletion of commensal microbiota and creating a microenvironment that allows the proliferation of pathogenic microbes (Yeoh et al., 2021). SARS-CoV-2 infections alter the stringent regulatory functions of commensal microorganisms of the gut, leading to the aberrant immune responses observed in COVID-19 patients. Patients with COVID-19 disease show a depletion of beneficial microbes, such as the Bifidobacterium genus, and an increase of opportunistic pathogens such as Streptococcus and Veillonella (Gu et al., 2020; de Oliveira et al., 2021).

Members of the Firmicutes and Bacteroidetes phyla are commensals that have directly affected SARS-CoV-2 pathogenicity and infection severity by their regulatory roles in the ACE2 gene (Zuo et al., 2020b). Members of the Bacteroidetes phylum are known to downregulate the expression of the ACE2 receptor. This correlation ultimately has a "protective" role in COVID-19 infections by minimizing the abundance of ACE2 receptors on intestinal cell surfaces, decreasing the interaction between the virion and the host cell. On the other hand, members of the Firmicutes phylum can upregulate ACE2 gene expression, leading to increased interaction between viral spike proteins and ACE2 receptors, resulting in a higher infection rate. Other commensals, such as Faecalibacterium prausnitzii, Eubacterium, Roseburia, and Lachnospiraceae taxa, have immune maintenance and anti-inflammatory properties. These commensals are associated with low infection severity and a low SARS-CoV-2 load in patient stool samples, suggesting that they play a role in combatting SARS-CoV-2 in the gut (Zuo et al., 2020b). These intestine-resident beneficial bacteria are depleted in patients with high infection severity (Xu et al., 2020). On the other hand, several opportunistic pathogens are enriched in the stool of COVID-19 patients, including Clostridium hathewayi, Actinomyces viscosus, and Bacteroides nordii (Gu et al., 2020; He et al., 2020). Together, the imbalance of the aforementioned gut microbiota results in the gastrointestinal symptoms prevalent in COVID-19 patients, and these microbiota perturbations persist even after the clearance of SARS-CoV-2 (Yeoh et al., 2021).

These data collectively indicate a direct correlation between the composition of the intestinal microbiota and SARS-CoV2 infection severity. Therefore, the microbial ecosystem before and during infection can help predict the severity of SARS$\mathrm{CoV}-2$ infection, and this can be used to mediate a patient's immune response to COVID-19. Therefore, this study explored the gut microbiota composition and functionality associated with SARS-CoV-2 infection in the United Arab Emirates.

\section{MATERIALS AND METHODS}

\section{Participants and Study Design}

This study involved 86 participants with previously confirmed COVID-19 infection and 57 healthy individuals as controls. SARS-CoV-2 infection was confirmed by two consecutive RTPCR tests targeting $\mathrm{N}$ and RdRp genes performed by accredited Abu Dhabi Health Services Company (SEHA) laboratories and RT-PCR tests targeting RdRp gene performed at the Center for Biotechnology at Khalifa University. At the time of the study, all COVID-19 cases completed their isolation period at a specialized facility in Abu Dhabi, United Arab Emirates. A COVID-19non-infected control cohort was recruited randomly as described before (Al Bataineh et al., 2021). All participants were provided with an information sheet and an explanation of the study objectives, design, and confidentiality. Volunteers signed the required consent form before proceeding with sample collection. The study was approved by the Abu Dhabi Health COVID-19 Research Ethics Committee (DOH/DQD/2020/538) and the SEHA Research Ethics Committee (SEHA-IRB-005). 


\section{Sample Collection and Handling Protocol}

A total of 86 nasal swab and fecal samples from clinically confirmed SARS-CoV-2-positive patients were collected at an isolation facility for COVID-19 patients in Abu Dhabi, United Arab Emirates, during the period of July-August 2020. The inclusion criteria for this study were being above 18 years old, able to give consent, and positive for the COVID-19 cohort. The exclusion criteria are being below 18 years old, unable to give consent, pregnant, and under antibiotics treatment. Swabs were collected in NUCLISWAB kits (SALUBRIS, Inc., Boston, MA, United States) by nurses. A sterile stool specimen container with an integrated collection spoon and collection instructions were provided to all subjects. Collection of 2-4 $\mathrm{g}$ of freshly passed stool in sterile containers was performed, and stool samples collected were transported on dry ice to the Center for Biotechnology at Khalifa University, Abu Dhabi, along with all vital metadata information for each patient's clinical severity. The specimens were stored immediately at $-80^{\circ} \mathrm{C}$.

\section{RNA Extraction and Quantification}

Viral RNA from nasal swabs was extracted using the MiracleAutoXT Automated Nucleic Acid Extraction System (iNtRON Biotechnology Inc., South Korea), and viral RNA from fecal samples was extracted using DNeasy PowerLyzer PowerSoil Kit (Qiagen Ltd., GmbH, Germany) following the manufacturer's instruction. Genes from the PrimerDesign RT-PCR COVID-19 detection kit (PrimerDesign Ltd.) were used for the quantification of the viral RNA in nasal swabs and fecal samples. The primers provided in the kit target the $R d R p$ gene. Internal extraction control primers were also provided to detect the exogenous source of the RNA template added during the extraction step. The PCRs were performed according to the manufacturer protocol using the Magnetic Induction Cycler (MiC) PCR Machine (Bio Molecular Systems, QLD, Australia).

\section{DNA Extraction}

Fecal samples were subjected to DNA extraction using the DNeasy PowerLyzer PowerSoil Kit (Qiagen Ltd., GmbH, Germany, catalog no. 12855-100) following the manufacturer's instruction (Qiagen Ltd.). DNA concentration and purity were evaluated by optical density using NanoDrop (Thermo Fisher Scientific, United States) at wavelengths of 230, 260, and $280 \mathrm{~nm}$, and DNA integrity was checked on $1 \%$ agarose gel electrophoresis stained with $0.5 \mathrm{mg} / \mathrm{ml}$ ethidium bromide.

\section{PCR Amplification and Sequencing of the V3 and V4 Hypervariable Regions of Bacterial 16S rRNA Genes}

16S rRNA sequencing for V3 and V4 hypervariable regions was carried out with extracted microbial DNA on the MiSeq platform. The viral load in fecal samples was parallel-examined and analyzed by qPCR methodology. For amplification of the V3 and V4 hypervariable regions of the bacterial 16S rRNA gene, primer pair sequences (Integrated DNA Technologies, United States) were used and generated a single amplicon of approximately $460 \mathrm{bp}$. The primer sequence design included overhang adapter sequences optimized for Illumina sequencing, which in turn were further processed by employing the $16 \mathrm{~S}$ Metagenomic Sequencing Library Preparation Protocol (Part no. 15044223 Rev. B, Illumina). MiSeq sequencing 16S V3 and V4 region-specific amplicons were further subjected to indexing PCR using Illumina Nextera XT index kit set A. The final libraries were purified and pooled according to the Illumina metagenomics workflow and were loaded on MiSeq using MiSeq V2 300 cycle reagent kit (Illumina, Inc., San Diego, CA, United States).

\section{Data Analysis}

The descriptive variables were verified using frequency analysis. The non-normal quantitative variables were presented as medians and interquartile ranges (IQRs), and the normal quantitative variables were presented as means and standard deviation (SD). Chi-square and Fisher's exact tests were used to study categorical variables. Independent-sample $t$-test or non-parametric Mann-Whitney $U$-tests were used to analyze continuous variables. Kruskal-Wallis or ANOVA tests were used to verify the association. Spearman correlations were calculated to establish bivariate relationships between viral load and relative abundance.

BCL files from sequencing were demultiplexed using Illumina's bcl2fastq tool. We subsequently describe the analysis pipeline for microbial communities based on QIIME 2, which underwent a paradigm shift from operational taxonomic units (OTUs) to amplicon sequence variants (ASVs), as the latter exhibit several advantages over the former (Langille et al., 2013; Callahan et al., 2017). After demultiplexing using the QIIME demux, sequence quality control and feature table construction have been performed with DADA2 (Supplementary Text). An average of 41,141 quality-filtered reads was generated per sample (50,665 and 26,575 for cases and control, respectively).

Visual summaries are generated with the QIIME commands feature-table summarize and feature-table tabulate-seqs. Subsequently, we generate a phylogeny for diversity analysis using QIIME phylogeny. The resulting phylogeny QIIME artifact, particularly the rooted phylogenetic tree, enables a range of diversity analyses using QIIME diversity commands. First, alpha diversity is determined in Shannon entropy, a commonly applied qualitative measure of community richness (Figure 1A). Next, a comprehensive diversity analysis is conducted with QIIME's command core-diversity-phylogenetic. It calculates a range of alpha- and beta-diversity metrics with and without using the previously generated phylogenetic tree. For example, weighted UniFrac analyzes how microbial communities cluster together based on the weighted phylogenetic branches shared between communities.

In addition, this step produces a range of output visualizations, such as three-dimensional principal coordinate analysis (PCoA) plots, shown in Figure 1B. Finally, we enable testing of associations between categorical metadata such as COVID-19-positive and COVID-19-non-infected with the help of QIIME command diversity alpha-group-significance, where community richness is expressed in terms of the Faith phylogenetic diversity. 

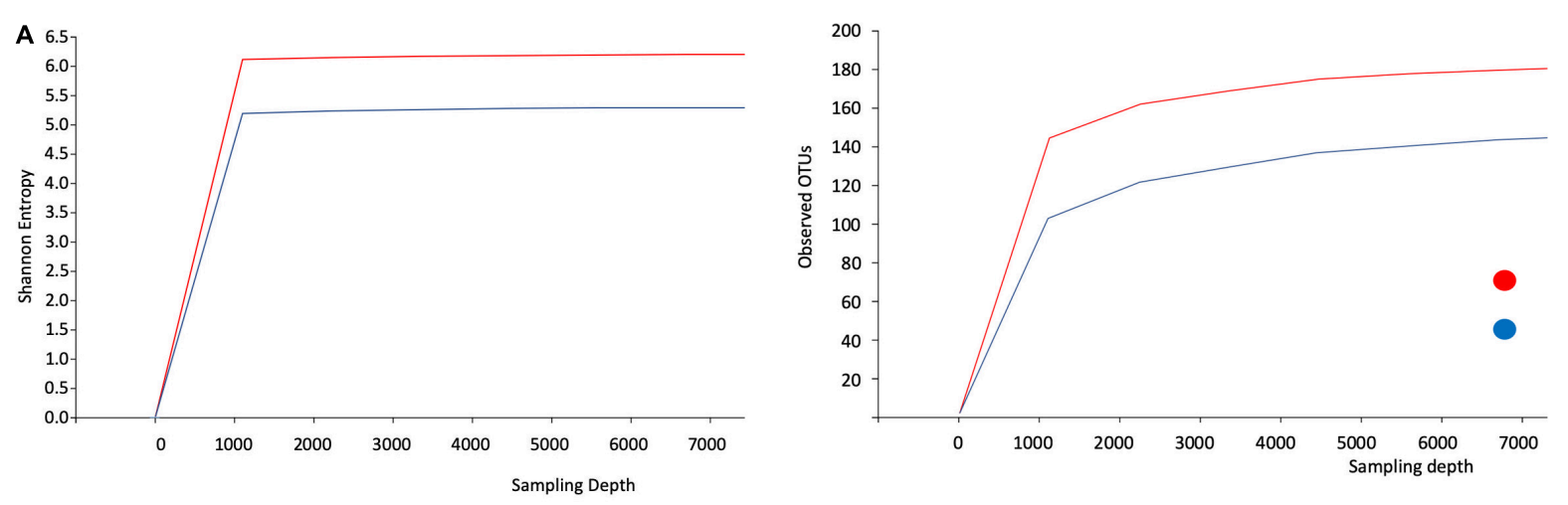

B

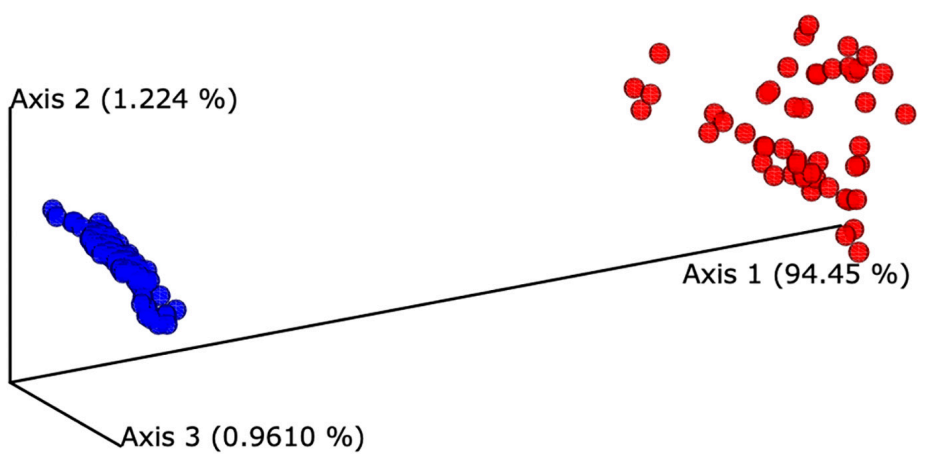

FIGURE 1 | Evaluation of the alpha- and beta-diversity of the gut microbiota of SARS-CoV-2-infected subjects. (A) Evaluation of alpha-diversity in the 143 analyzed samples. The outlined graphs report the average rarefaction curves based on Shannon entropy and raw count of features increasing sequencing depth of SARS-CoV-2-infected and SARS-CoV-2-non-infected samples. (B) Evaluation of beta-diversity. The panel shows the predicted principal coordinate analysis (PCoA) plot based on weighted UniFrac distances. SARS-CoV-2-infected and SARS-CoV-2-non-infected sample datasets are colored in red and blue, respectively.

The QIIME output was analyzed using the DESeq2 package from BioconductR using custom-written scripts in the $R$ programming language (3.6.1) (Love et al., 2014; Huber et al., 2015). The standard workflow of running estimateSizeFactors, estimateDispersions, and nbinomWaldTest was used to input the raw (un-normalized) counts. Adjusted $p$-values reflect $\mathrm{BH}$ false discovery rates (Benjamini and Hochberg, 1995). Results adjusting for gender and age group were obtained by using a likelihood ratio instead of the Wald test in the DESeq2 calculations. We also conducted a phylogenetic investigation of communities by reconstructing unobserved state (PICRUSt) functional analyses based on $16 \mathrm{~S}$ rRNA gene abundance profiles. The PICRUSt un-normalized output was also analyzed using the standard DESeq2 workflow. PICRUSt not only estimates full metagenomes from 16S rRNA data but also estimates for each sample what OTU contributed to each predicted gene (metagenome_contribution.py). We perform this analysis for selected pathways of interest by first identifying the associations of the respective genes to pathways and then aggregating contribution in terms of the phylogenetic ranks family and genus. This step accumulates positive and negative samples. All script files for executing this workflow can be found at https://github.com/sffeng/microbiome_covid_uae. All QIIME and PICRUSt commands are documented in the Supplementary Material. The R environment was utilized for visualization tools (Wickham, 2009).

\section{RESULTS}

\section{Gut Microbiota of Severe Acute Respiratory Syndrome Coronavirus 2-Infected Subjects Show Compositional Differences From Severe Acute Respiratory Syndrome Coronavirus 2-Non-infected Subjects}

We explored the compositional variation of the gut microbiome among 86 COVID-19-positive and 57 COVID-19-non-infected individuals from the United Arab Emirates. Relevant clinical features are shown in Table 1. SARS-CoV-2-infected subjects demonstrate a significant gender difference $(p$-value $<0.001)$, age ( $p$-value $<0.001$ ), probiotics use ( $p$-value $<0.001$ ), ethnicity $(p$-value $=0.019)$, and fiber intake $(p$-value $=0.011)$ compared to SARS-CoV-2-non-infected subjects (Table 1). However, both groups showed no significant difference in BMI $(p$-value $=0.507)$ (Healey et al., 2016).

We examined the taxonomic composition generated from high-quality reads and classified using Silva as the reference database. We aggregated ASVs into each taxonomic rank and plotted the relative abundance (Supplementary Text 2 and Supplementary Figure 1). The taxonomic plot shows a clear distinction between positive and negative samples. This difference is mainly attributable to the high presence 
TABLE 1 | Demographic characteristics of participants by case and control group.

\begin{tabular}{lccc}
\hline & $\begin{array}{c}\text { CovID-19 Cases } \\
(\boldsymbol{n}=\mathbf{8 6}), \boldsymbol{n}(\%)\end{array}$ & $\begin{array}{c}\text { Controls }(\boldsymbol{n}=\mathbf{5 7}), \\
\boldsymbol{n}(\%)\end{array}$ & $\boldsymbol{p}$-value \\
\hline Gender & & & \\
Male & $52(60.5 \%)$ & $14(24.6 \%)$ & $<0.001$ \\
Female & $34(39.5 \%)$ & $43(75.4 \%)$ & \\
Age & & & \\
$<25$ & $24(27.2 \%)$ & $13(22.8 \%)$ & $<0.001$ \\
$26-35$ & $27(31.4 \%)$ & $10(17.5 \%)$ & \\
$36-51$ & $25(29.1 \%)$ & $9(15.8 \%)$ & \\
$>52$ & $10(11.6 \%)$ & $25(43.9 \%)$ & \\
BMI & & & \\
$\leq 18.50$ & $3(3.7 \%)$ & $2(3.5 \%)$ & 0.507 \\
18.51-24.99 & $24(29.3 \%)$ & $18(31.6 \%)$ & \\
$25.00-29.99$ & $34(41.5 \%)$ & $17(29.8 \%)$ & \\
$\geq 30.00$ & $21(25.6 \%)$ & $20(35.1 \%)$ & \\
Region of origin & & & \\
Middle Eastern & $75(87.2 \%)$ & $57(100.0 \%)$ & 0.019 \\
Asian & $9(10.5 \%)$ & $0(0.0 \%)$ & \\
African & $2(2.3 \%)$ & $0(0.0 \%)$ & \\
Use of probiotics & & & \\
Yes & $23(26.7 \%)$ & $32(56.1 \%)$ & $<0.001$ \\
No & $63(73.3 \%)$ & $25(43.9 \%)$ & \\
Fiber & & & \\
High-fiber diet & $27(31.4 \%)$ & & \\
Low-fiber diet & $59(68.6 \%)$ & & \\
\hline & & & \\
\hline
\end{tabular}

of the Lachnospiraceae genus in positive samples (shown in purple, top bar contribution). Furthermore, Blautia and Faecalibacterium appear dominant in positive samples, whereas Prevotella features a substantial part of the negative samples. Next, we evaluated averaged alpha-diversity for the 143 subjects. Analysis of the averaged rarefaction curves based on Shannon entropy and observed feature count at increasing sequencing depth displayed that both curves plateau, suggesting adequate coverage for most of the biodiversity in the samples. SARSCoV-2-infected samples show an average increased level of gut microbiota complexity compared to SARS-CoV-2-non-infected samples (Figure 1A).

Alpha rarefaction (measuring both Shannon entropy and observed features) shows that each rarefaction depth has a substantially larger diversity for SARS-CoV-2-infected samples, in terms of both Shannon entropy and observed OTUs. As most samples in this study contain at least 11,000 samples, we chose this number as the maximum sampling depth during rarefaction. In addition, the rarefaction curve's plateau shapes strongly indicate that the sequencing depths are adequate and give a true reflection of representative community composition.

We also evaluated the compositional diversity among samples and the beta-diversity of the gut microbiome. All samples are expressed as ASV feature tables, for which we calculate all-against-all distance matrices, where distances between samples are expressed in terms of weighted UniFrac. We subsequently subject the distance matrix to PCoA (Figure 1B).

\section{Evaluation of Bacterial Relative} Abundance and Prevalence Between Severe Acute Respiratory Syndrome Coronavirus 2-Infected and Severe Acute Respiratory Syndrome Coronavirus 2-Non-infected Individuals

To explore the alterations in bacterial richness and diversity due to SARS-CoV-2 infection, as shown in Figure 1, we adjusted for gender and age group. We conducted a likelihood ratio test to compare the average relative abundance and total prevalence between both groups. The comparison between both datasets revealed that SARS-CoV-2-infected individuals have statistically significant enrichment of Blautia (relative abundance 13.93\% in cases vs. $4.87 \%$ in control, on average, $p$-value $=9.22 \times 10^{-18}$ ), Faecalibacterium (relative abundance $12.57 \%$ vs. $4.80 \%$, $p$-value $\left.=1.72 \times 10^{-6}\right)$, Streptococcus (relative abundance $2.93 \%$ vs. $0.84 \%, p$-value $\left.=1.24 \times 10^{-6}\right)$, among others $($ Figure 2$)$. The following bacterial genera demonstrated a depletion in SARS$\mathrm{CoV}$-2-infected individuals: Intestinibacter (relative abundance $0 \%$ vs. $0.23 \%, p$-value $\left.=4.06 \times 10^{-90}\right)$, Enterorhabdus (relative abundance $0 \%$ vs. $0.10 \%$, $p$-value $\left.=9.50 \times 10^{-50}\right)$, Anaerostipes (relative abundance $0 \%$ vs. $1.47 \%, p$-value $=1.75 \times 10^{-230}$ ), Bifidobacterium (relative abundance $2.86 \%$ vs. $7.22 \%$, $p$-value $=4.50 \times 10^{-8}$ ), Bacteroides (relative abundance $8.23 \%$ vs. $15.27 \%$, $p$-value $\left.=5.67 \times 10^{-8}\right)$, and Prevotella $($ relative abundance $3.07 \%$ vs. $11.37 \%$, $p$-value $=1.59 \times 10^{-3}$ ).

To further investigate the association between the gut microbiomes and COVID-19 viral load, we performed Spearman's rank-order correlation between the viral load in nasal and stool samples to the relative abundance of the gut microbiome, which demonstrated a lack of significant association (Supplementary Table 3). When categorizing viral load into a bivariate categorical group, where a high viral load is defined as the viral load (genome copied/ $\mu$ l) being above the median (IQR) cycle threshold value for the detection of SARS-CoV-2 using quantitative reverse-transcription PCR (median: 31.76). There was no significant association between high vs. low SARS-CoV-2 viral load on any of the bacterial microbiome (Supplementary Table 4) when running an independent $T$-test to measure association $(p<0.05)$.

\section{Functional Characterization of Severe Acute Respiratory Syndrome Coronavirus 2-Infected and Severe Acute Respiratory Syndrome Coronavirus 2-Non-infected Microbiomes Based on Phylogenetic Investigation of Communities by Reconstructing Unobserved State Analyses of 16S rRNA} Gene Profiles

To obtain a deeper insight into the possible functional contributions of the gut microbiome on individuals with COVID-19, we conducted PICRUSt prediction analyses based on $16 \mathrm{~S}$ rRNA gene abundance profiles. Functional profiling revealed 


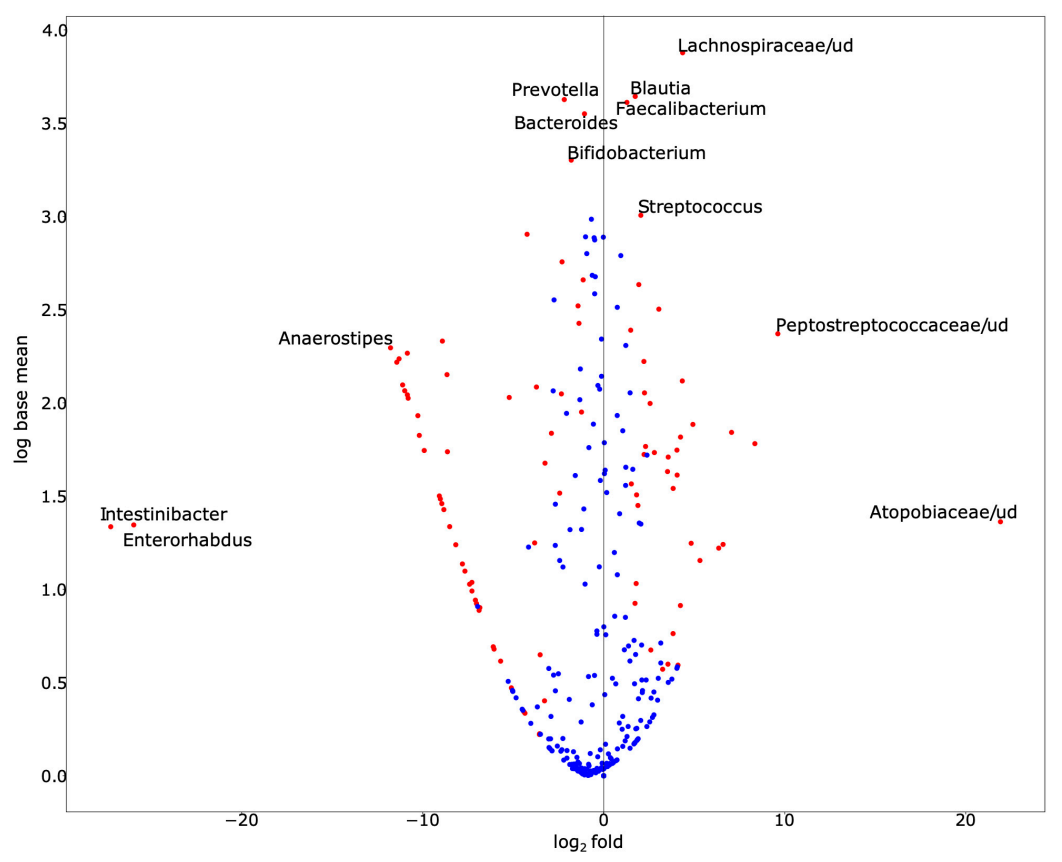

FIGURE 2 | Exploration of bacterial abundance and prevalence in SARS-CoV-2-infected and SARS-CoV-2-non-infected control groups. The volcano scatter plot of base mean abundance between both groups shows the log2 change between infected vs. non-infected samples on the horizontal axis. The vertical axis shows the abundance. Red dots indicate statistical significance after multiple testing corrections at the significance level 0.05. This figure was produced by DESeq2. While we observe that many species are significantly different, the labeled ones of interest demonstrate either especially large effect sizes or large abundances.

Supplementary Table 1 demonstrates the base mean, fold change, effect size, and adjusted $p$-value (for gender and age-group via a likelihood ratio test) of each associated bacterial genus. Supplementary Table 2 demonstrates the total prevalence and average relative abundance of each group.

significant overall differences in metabolic potential between both groups. Over 250 functional pathways were reported to be significantly upregulated or downregulated in relation to COVID-19 status (Supplementary Table 4). We further categorized these pathways and reported them in a bar plot of fold changes related to SARS-CoV-2 infection (Figure 3).

We identified significant enrichment of metabolic pathways implicated in the following: fatty acid and lipid biosynthesis and degradation [Figure 3A; ether lipid metabolism $(p=2.52$ $\left.\times 10^{-9}\right)$, lipid metabolism $\left(p=5.46 \times 10^{-10}\right)$, bisphenol degradation $\left(p=2.89 \times 10^{-5}\right)$, glycolipid metabolism $(p=2.47$ $\left.\times 10^{-12}\right)$, and glycerophospholipid metabolism $(p=1.79 \times$ $\left.10^{-12}\right)$, amino acid and protein metabolism and degradation [Figure 3B; linoleic acid metabolism $\left(p=1.84 \times 10^{-6}\right)$; lipid metabolism $\left(p=2.31 \times 10^{-9}\right)$; lysine biosynthesis $(p=5.65 \times$ $\left.10^{-17}\right)$; and valine, leucine, and isoleucine biosynthesis $(p=$ $\left.4.41 \times 10^{-6}\right)$ ], xenobiotic metabolism [Figure 3C; tetracycline biosynthesis $\left(p=2.12 \times 10^{-13}\right)$, bacterial chemotaxis $(p=9.26$ $\left.\times 10^{-7}\right)$, beta-lactam resistance $\left(p=6.56 \times 10^{-5}\right)$, and xylene degradation $\left.\left(p=1.91 \times 10^{-3}\right)\right]$, carbohydrate metabolism and degradation [Figure 3D; synthesis and degradation of ketone bodies $\left(p=4.72 \times 10^{-5}\right)$, fructose and mannose metabolism $\left(p=1.11 \times 10^{-4}\right)$, pentose phosphate pathway $(p=1.98 \times$ $\left.10^{-7}\right)$ ], and others [Figure 3F; pathogenic Escherichia coli infection $\left.\left(p=6.56 \times 10^{-6}\right)\right]$. Furthermore, we identified bacterial genera and metabolic pathways correlated with statistically significant differences in abundance between groups.
Therefore, we estimate the major contributing families/genera to those four pathways: Enterobacteriaceae/Parabacteroides, Streptococcaceae/Streptococcus, Lachnospiraceae/Dorea, and Lachnospiraceae/Blautia. Furthermore, the associated genes for "pathogenic E. coli infection," prominent in positive samples, were mainly contributed $(68.3 \%)$ by Enterobacteriaceae (Supplementary Table 6).

\section{DISCUSSION}

The study of COVID-19 relationship with the human microbiota is a rapidly emerging area of research, but a complete characterization of the gut microbiota connection with COVID19 pathogenesis is still unclear (Zuo et al., 2020b; RosasSalazar et al., 2021; Xu et al., 2021). To the best of our knowledge, this is the first report on the emerging COVID-19 interaction with the gut microbiota among Middle Eastern populations. This study determined changes in the gut microbiota composition and species abundance in SARSCoV-2-infected individuals. First, we described the bacterial taxonomic diversity among SARS-CoV-2-infected and SARS$\mathrm{CoV}-2$-non-infected groups. SARS-CoV-2 infection exerts a substantial effect on bacterial richness and complexity, as shown in Figure 1A. One of the most intriguing findings was the notable compositional diversity difference between groups; COVID-19 patients clustered uniformly but shifted away from the control, 


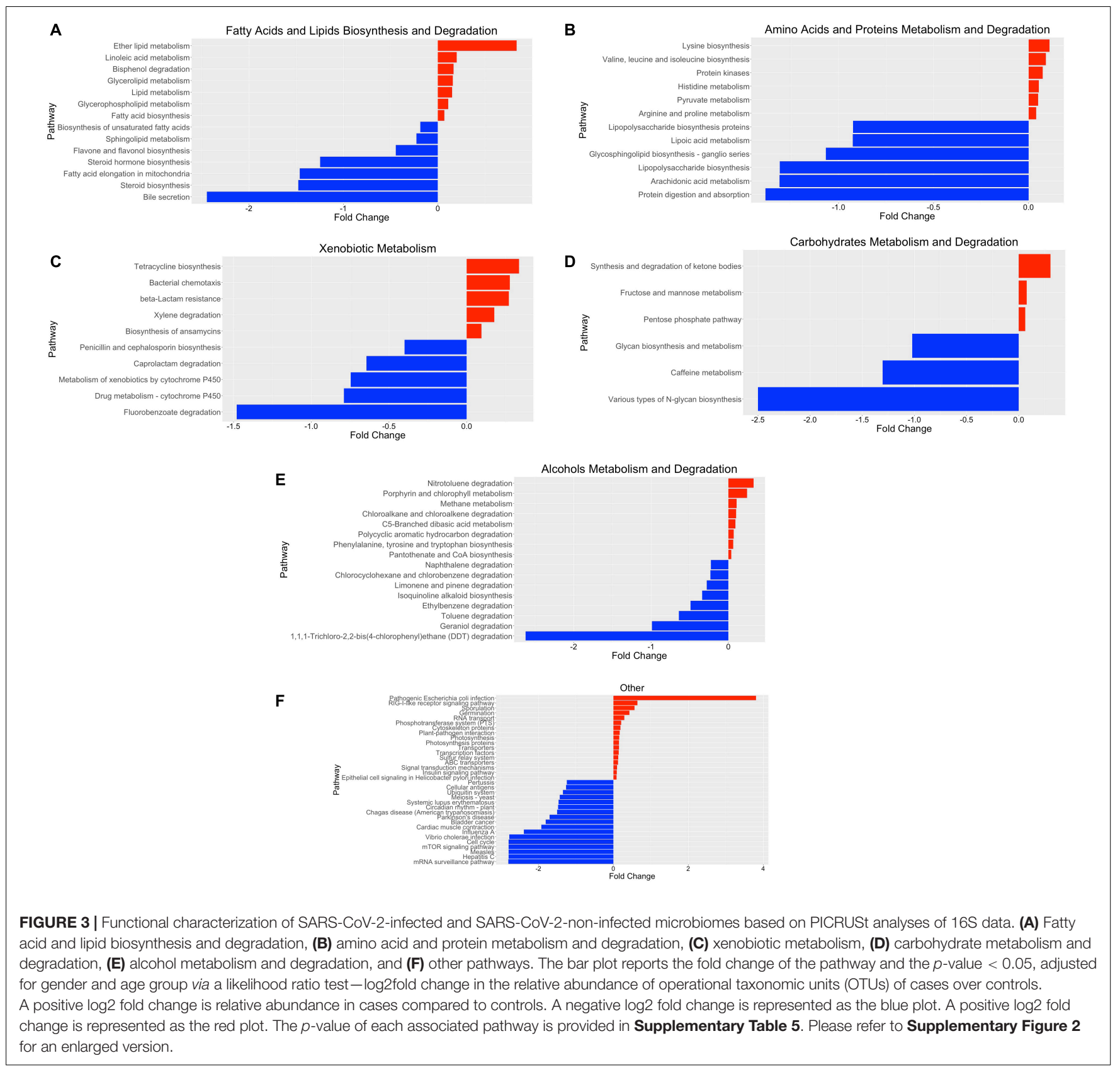

as shown in Figure 1B. Perhaps this extreme clustering shift can be attributed to a significant gender and age difference between both groups, as shown in Supplementary Table 1. The control group was older and had a 3:1 female-to-male ratio. Numerous studies have revealed gender differences in human gut microbiota. A 2016 large-cohort study with two extensively phenotyped independent groups determined that gender significantly correlates with the overall microbiome variation (Falony et al., 2016). Furthermore, gut microbiota changes with age, displaying a distinct inflammatory profile with increased susceptibility to infections. We determined significant enrichment of Lachnospiraceae among the COVID-19 patient group, which plays an essential role in gut barrier function and immune tolerance, especially against local inflammation in a young age group (DeJong et al., 2020).

Recent studies showed gut dysbiosis with reduced bacterial richness and diversity among hospitalized COVID-19 patients (Zuo et al., 2020b; Xu et al., 2021). On the contrary, we observed higher bacterial richness in the SARS-CoV-2-infected group. Therefore, we hypothesized that the genera of gut bacterial enrichment might correlate with the observed minor gastrointestinal signs and symptoms among SARS-CoV2-infected subjects. Furthermore, we determined a higher relative abundance of anti-inflammatory bacteria such as Faecalibacterium and Bacteroides in the SARS-CoV-2-infected group (Zuo et al., 2020a,b). Furthermore, we also showed a higher 
relative abundance of the highly beneficial butyrate-producing bacteria, such as Faecalibacterium, Anaerostipes, Lachnospiraceae, and Blautia in the SARS-CoV-2-infected group (Riviere et al., 2016; Gu et al., 2020; Zuo et al., 2020b). Altogether, perhaps the gut symbiotic response plays a significant role in counteracting COVID-19 dysregulated immune response, restoring homeostasis, and subsequently reducing COVID-19 pathogenesis and disease manifestations (Bui et al., 2019). Conversely, we still noticed a significantly higher relative abundance of some pathogenic and pro-inflammatory bacteria, consistent with previous literature, such as Streptococcus and Prevotella spp., which may have influenced the initial COVID-19 presentation in our cohort (Iljazovic et al., 2020). These results here were adjusted for gender and age group via a likelihood ratio test as mentioned previously.

We attempted to explore the functional contribution of the gut microbiota in COVID-19 pathogenesis, which may become useful in predicting new microbial biomarkers for COVID-19 diagnostic and management strategies. PICRUSt functional analyses of the 16S rRNA abundance data showed a substantial difference in metabolic capacity between the SARS-CoV-2-infected and SARS-CoV-2-non-infected groups, as shown in Figure 3. Furthermore, we identified several significantly abundant pathways involved in lipid, amino acid, carbohydrate, and xenobiotic metabolism, among others, in the SARS-CoV-2-infected group. Lipids play various critical cellular functions and are implicated in several stages during viral replication, and it was found to be directly linked to coronavirus spread and multiplication (Yan et al., 2019). Here, we discovered an enrichment of linoleic acid metabolism, ether lipid metabolism, bisphenol degradation, glycerolipid metabolism, and glycerophospholipid metabolism in the SARS-CoV-2-infected group. Interestingly, the correlations between coronavirus-induced modifications of host lipid metabolism and bioavailability of plasmalogens (vinyl ether glycerophospholipids) in host cells are crucial for SARS-CoV-2 entry and replication (Schloer et al., 2019, 2021; Deng and Angelova, 2021). For example, studies have shown that host ether lipid metabolism and plasmalogens were essential and further enhanced during viral infections (Liu et al., 2011; Martin-Acebes et al., 2014; Tanner et al., 2014). Remarkably, our PICRUSt prediction confirmed that this might also apply to SARS-CoV-2 infection, as shown in Figure 3A (Deng and Angelova, 2021). Therefore, we further performed analysis for these pathways to identify the individual bacterial gene contribution toward these pathways' enrichment (Supplementary Table 3). Interestingly, we determined a significant contribution of Parabacteroides, Streptococcus, Dorea, and Blautia genera toward these lipid metabolism pathways. Previous studies correlated these genera with short-chain fatty acid production, metabolic dysbiosis reduction, and anti-inflammatory activity increase (Jenq et al., 2015; Garcia-Mantrana et al., 2018; Lee et al., 2019; Wang et al., 2019; Todorov et al., 2020). Moreover, several other studies have demonstrated how microbial lipids alter circulating host cholesterol and sphingolipid concentrations, thus impacting human lipid homeostasis (Lamichhane et al., 2021; Nanda and Ghosh, 2021). For example, a study found that tocilizumab treatment resulted in host lipid and metabolic alterations due to SARS-CoV-2 infection (Meoni et al., 2021). Another study found a significant correlation between drug-induced phospholipidosis and inhibition of SARS-CoV-2 replication in cells (Tummino et al., 2021).

Furthermore, Winkler et al. determined that the gut microbiome Clostridium scindens supports antiviral protection through a bile acid-IFN signaling axis. Likewise, Parabacteroides was found to alleviate obesity and metabolic dysfunctions via the production of succinate and bile acids (Wang et al., 2019; Winkler et al., 2020). Therefore, we hypothesize a direct role for the aforementioned genus to ameliorate SARS-CoV-2 entry to and replication in the host cell and to reduce COVID-19 severity, as evident by the mild clinical manifestations in the infected group. The predicted linkage to particular gut microbiota members may prove helpful as a microbial biomarker to provide new tools for COVID-19 management strategies.

We also observed enrichment of pathways involved in amino acid metabolism. A recent metabolomics analysis study proposed an essential role for branched-chain amino acids during hypoxic conditions associated with COVID-19 via an $\alpha$-keto-acid oxidase mechanism, underscoring a plausible link to gut microbiota supplementation of amino acid during SARS-CoV-2 infection to mitigate disease severity (Paez-Franco et al., 2021). Furthermore, we also noted a shift from the liver cytochrome P450mediated drug metabolism toward other functions, as shown in Figure 3C. Chemokines and cytokines play a significant role in COVID-19 immunopathology, as they are the underlying cause for exacerbated immune response leading to cytokine storm (Henderson et al., 2020). Cytokine increase and inflammation during SARS-CoV-2 and other viral infections have been shown to suppress cytochrome P450 enzymes, thereby resulting in hepatic clearance of xenobiotics (Deb and Arrighi, 2021).

Interestingly, we also noted a robust upregulation of the pathogenic $E$. coli infection pathway function among the SARSCoV-2-infected group, as shown in Figure 3F. COVID-19 coinfection with pathogenic and opportunistic bacteria is well established in literature (Calcagno et al., 2021). The associated genes for the pathogenic E. coli infection pathway among positive samples were mainly contributed (68.3\%) by Enterobacteriaceae, a large family of Gram-negative bacteria, including E. coli.

We want to mention that participants in this study demonstrated significant age, gender, and probiotic use differences, as shown in Supplementary Table 1. Still, they share similar lifestyle and dietary habits such as dietary fiber intake in both groups. Future longitudinal cohorts would be more beneficial to understand the temporal relationship between SARS-CoV-2 susceptibility as well as severity and the compositional changes of the gut microbiome. Due to the heterogeneous nature of the SARS-CoV-2 phenotype, affected patients were not classified based on symptom severity and hence lacked stratified analysis.

In conclusion, our data report a significant compositional and functional shift in the gut microbiota of COVID-19 patients. We observed an increased relative abundance of beneficial bacteria based on the relative ratio changes of significant taxa between SARS-CoV-2-infected and SARS-CoV-2-non-infected 
individuals. The investigated bacterial taxonomic profiles suggested a biological shift toward anti-inflammation in the SARS-CoV-2-infected group that may explain the mild COVID-19 sign and symptoms in this group. Furthermore, SARS-CoV-2-infected individuals exhibited a higher density of bacterial genes enriched in pathways directly involved in lipid metabolism, primarily Parabacteroides, Streptococcus, Dorea, and Blautia genera. We also showed that the compositional changes in the gut microbiota were not affected by gender and age. Altogether, our findings suggest a putative role for the gut microbiota in protecting against SARS-CoV-2 infection. However, these findings should be followed by additional validation studies on larger cohorts involving populations with different environmental conditions and genetic backgrounds. The identified bacterial genera can most likely provide screening biomarkers to predict COVID-19 pathogenesis and better manage disease severity in the era of the COVID-19 pandemic with increased demands on healthcare.

\section{MEMBERS OF THE UAE COVID-19 COLLABORATIVE PARTNERSHIP}

Juan Acuna, Eman Alefishat, Ernesto Damiani, and Abdulrahim Sajini (Khalifa University, Abu Dhabi, United Arab Emirates); Bassam Ali (United Arab Emirates University, Al Ain, United Arab Emirates); Hiba Alhumaidan, Hala Imambabaccus, Amirtharaj Francis, and Stefan Weber (Sheikh Khalifa Medical City and SEHA, Abu Dhabi, United Arab Emirates); Rabih Halwani and Rifat Akram Hamoudi (University of Sharjah, Sharjah, United Arab Emirates); Abdulmajeed Alkhajeh, Laila Salameh, and Bassam H. Mahboub (Dubai Health Authority, Dubai, United Arab Emirates); and Braulio Peramo (Al Ain Fertility Center, Al Ain, United Arab Emirates).

\section{DATA AVAILABILITY STATEMENT}

The data that support the findings of this study has been deposited at Gene Expression Omnibus (GEO) database with accession number (GSE185406).

\section{ETHICS STATEMENT}

The studies involving human participants were reviewed and approved by the Abu Dhabi Health COVID-19 Research Ethics Committee (DOH/DQD/2020/538) and the SEHA Research Ethics committee (SEHA-IRB-005). The patients/participants provided their written informed consent to participate in this study.

\section{AUTHOR CONTRIBUTIONS}

HA and GT conceived the project and established the administrative framework for the "UAE COVID-19 Collaborative Partnership (CCP)" to allow for a multicentered approach to study the contribution of the SARS-CoV-2 virus and its human host to the COVID-19 disease in the United Arab Emirates. To provide the opportunity to examine the role of the patients' microbiome described in this manuscript, HA worked with MA, HK, MU, and NA to establish the protocols for patient recruitment and data collection for the study. They managed the sample collection process from consenting patients, managed the delivery of samples to the laboratory, and arranged for the experiments to be carried out. MA, MAK, and HA generated the initial research questions to study the microbiome data and initiated the first draft of the manuscript from data arising from microbiome sequencing performed by FW and first-pass analysis performed by $\mathrm{AH}$. The microbiome analysis pipeline conducted by $\mathrm{AH}$ required trimming and application of quality control (QC) steps to the raw microbiome sequence data to allow the extraction of high-quality sequence variants, which subsequently led to the predicted metagenomes. Along with primary contributions from SF, MD, and MM, data analysis and presentation were further refined. As more details became available, more specific research questions were posed by $\mathrm{AH}$, discussed during CCP meetings, and following extensive discussion, relevant points were accepted for inclusion into the manuscript. MK, AA, GT, MA, AH, MAK, and HA provided critical review during manuscript preparation. MM managed the circulation of the different versions of the manuscript and collated comments from all parties. Authors on the primary list contributed some part to the data interpretation or critically reviewed the manuscript and provided final approval for the manuscript to be submitted. All authors contributed to the article and approved the submitted version.

\section{FUNDING}

The project was funded by internal funds provided by Khalifa University, awarded to HA (grant code CPRA-2020-004), and funded by the University of Sharjah, awarded to MA (grant codes: 1901090253 and 1701090226).

\section{ACKNOWLEDGMENTS}

We thank the study participants for their generosity in providing samples to advance our understanding of COVID-19 infection. We acknowledge the assistance of the healthcare workers at the frontline of the COVID-19 pandemic. Special thanks to Mohamed Almarei and Mohamed Alhashami during patient recruitment; without their help, this study would not have been possible. We are also grateful to Zainab Alhalwachi, who assisted in processing the samples in the laboratory.

\section{SUPPLEMENTARY MATERIAL}

The Supplementary Material for this article can be found online at: https://www.frontiersin.org/articles/10.3389/fmicb.2021. 761067/full\#supplementary-material 


\section{REFERENCES}

Al Bataineh, M. T., Dash, N. R., Bel Lassen, P., Banimfreg, B. H., Nada, A. M., Belda, E., et al. (2021). Publisher correction: revealing links between gut microbiome and its fungal community in type 2 diabetes mellitus among emirati subjects: a pilot study. Sci. Rep. 11:6153. doi: 10.1038/s41598-021-85464-3

Benjamini, Y., and Hochberg, Y. (1995). Controlling the false discovery rate: a practical and powerful approach to multiple testing. J. R. Stat. Soc. Ser. B 57, 289-300. doi: 10.2307/2346101

Bui, T. P. N., Schols, H. A., Jonathan, M., Stams, A. J. M., de Vos, W. M., and Plugge, C. M. (2019). Mutual metabolic interactions in co-cultures of the intestinal anaerostipes rhamnosivorans with an acetogen, methanogen, or pectin-degrader affecting butyrate production. Front. Microbiol. 10:2449. doi: 10.3389/fmicb.2019.02449

Calcagno, A., Ghisetti, V., Burdino, E., Trunfio, M., Allice, T., Boglione, L., et al. (2021). Co-infection with other respiratory pathogens in COVID-19 patients. Clin. Microbiol. Infect. 27, 297-298. doi: 10.1016/j.cmi.2020.08.012

Callahan, B. J., McMurdie, P. J., and Holmes, S. P. (2017). Exact sequence variants should replace operational taxonomic units in marker-gene data analysis. ISME J. 11, 2639-2643. doi: 10.1038/ismej.2017.119

de Oliveira, G. L. V., Oliveira, C. N. S., Pinzan, C. F., de Salis, L. V. V., and Cardoso, C. R. B. (2021). Microbiota modulation of the gut-lung Axis in COVID-19. Front. Immunol. 12:635471. doi: 10.3389/fimmu.2021.635471

Deb, S., and Arrighi, S. (2021). Potential effects of COVID-19 on Cytochrome P450-mediated drug metabolism and disposition in infected patients. Eur. J. Drug Metab. Pharmacokinetics 46, 185-203. doi: 10.1007/s13318-020-00668-8

DeJong, E. N., Surette, M. G., and Bowdish, D. M. E. (2020). The gut microbiota and unhealthy aging: disentangling cause from consequence. Cell Host Microbe 28, 180-189. doi: 10.1016/j.chom.2020.07.013

Deng, Y., and Angelova, A. (2021). Coronavirus-induced host cubic membranes and lipid-related antiviral therapies: a focus on bioactive plasmalogens. Front. Cell Dev. Biol. 9:630242. doi: 10.3389/fcell.2021.630242

Falony, G., Joossens, M., Vieira-Silva, S., Wang, J., Darzi, Y., Faust, K., et al. (2016). Population-level analysis of gut microbiome variation. Science 352, 560-564.

Garcia-Mantrana, I., Selma-Royo, M., Alcantara, C., and Collado, M. C. (2018). Shifts on gut microbiota associated to mediterranean diet adherence and specific dietary intakes on general adult population. Front. Microbiol. 9:890. doi: 10.3389/fmicb.2018.00890

Gu, S., Chen, Y., Wu, Z., Chen, Y., Gao, H., Lv, L., et al. (2020). Alterations of the gut microbiota in patients with Coronavirus Disease 2019 or H1N1 influenza. Clin. Infect. Dis. 71, 2669-2678. doi: 10.1093/cid/ciaa709

He, L.-H., Ren, L.-F., Li, J.-F., Wu, Y.-N., Li, X., and Zhang, L. (2020). Intestinal flora as a potential strategy to fight SARS-CoV-2 infection. Front. Microbiol. 11:1388. doi: 10.3389/fmicb.2020.01388

Healey, G., Brough, L., Murphy, R., Hedderley, D., Butts, C., and Coad, J. (2016). Validity and reproducibility of a habitual dietary fibre intake short food frequency questionnaire. Nutrients 8:558. doi: 10.3390/nu8090558

Henderson, L. A., Canna, S. W., Schulert, G. S., Volpi, S., Lee, P. Y., Kernan, K. F., et al. (2020). On the alert for cytokine storm: immunopathology in COVID-19. Arthritis Rheumatol. 72, 1059-1063. doi: 10.1002/art.41285

Hoffmann, M., Kleine-Weber, H., Schroeder, S., Krüger, N., Herrler, T., Erichsen, S., et al. (2020). SARS-CoV-2 cell entry depends on ACE2 and TMPRSS2 and is blocked by a clinically proven protease inhibitor. Cell 181, 271.e8-280.e8. doi: 10.1016/j.cell.2020.02.052

Huber, W., Carey, V. J., Gentleman, R., Anders, S., Carlson, M., Carvalho, B. S., et al. (2015). Orchestrating high-throughput genomic analysis with Bioconductor. Nat. Methods 12, 115-121. doi: 10.1038/nmeth.3252

Iljazovic, A., Roy, U., Gálvez, E. J., Lesker, T. R., Zhao, B., Gronow, A., et al. (2020). Perturbation of the gut microbiome by Prevotella spp. enhances host susceptibility to mucosal inflammation. Mucosal Immunol. 14, 1-12.

Jenq, R. R., Taur, Y., Devlin, S. M., Ponce, D. M., Goldberg, J. D., Ahr, K. F., et al. (2015). Intestinal blautia is associated with reduced death from graft-versushost disease. Biol. Blood Marrow Trans. 21, 1373-1383. doi: 10.1016/j.bbmt. 2015.04.016

Lamichhane, S., Sen, P., Alves, M. A., Ribeiro, H. C., Raunioniemi, P., Hyötyläinen, T., et al. (2021). Linking gut microbiome and lipid metabolism: moving beyond associations. Metabolites 11:55.
Langille, M. G., Zaneveld, J., Caporaso, J. G., McDonald, D., Knights, D., Reyes, J. A., et al. (2013). Predictive functional profiling of microbial communities using 16S rRNA marker gene sequences. Nat. Biotechnol. 31, 814-821. doi: $10.1038 /$ nbt. 2676

Lee, H.-C., Yu, S.-C., Lo, Y.-C., Lin, I. H., Tung, T.-H., and Huang, S.-Y. (2019). A high linoleic acid diet exacerbates metabolic responses and gut microbiota dysbiosis in obese rats with diabetes mellitus. Food Funct. 10, 786-798. doi: 10.1039/C8FO02423E

Lee, J. J., Kopetz, S., Vilar, E., Shen, J. P., Chen, K., and Maitra, A. (2020). Relative abundance of SARS-CoV-2 entry genes in the enterocytes of the lower gastrointestinal tract. Genes 11:645. doi: 10.3390/genes11060645

Li, M. Y., Li, L., Zhang, Y., and Wang, X. S. (2020). Expression of the SARS-CoV-2 cell receptor gene ACE2 in a wide variety of human tissues. Infect. Dis. Poverty 9:45. doi: 10.1186/s40249-020-00662-x

Liu, S. T., Sharon-Friling, R., Ivanova, P., Milne, S. B., Myers, D. S., Rabinowitz, J. D., et al. (2011). Synaptic vesicle-like lipidome of human cytomegalovirus virions reveals a role for SNARE machinery in virion egress. Proc. Natl. Acad. Sci. U.S.A. 108, 12869-12874. doi: 10.1073/pnas.1109796108

Love, M. I., Huber, W., and Anders, S. (2014). Moderated estimation of fold change and dispersion for RNA-seq data with DESeq2. Genome Biol. 15:550. doi: 10.1186/s13059-014-0550-8

Martin-Acebes, M. A., Merino-Ramos, T., Blazquez, A. B., Casas, J., EscribanoRomero, E., Sobrino, F., et al. (2014). The composition of West Nile virus lipid envelope unveils a role of sphingolipid metabolism in flavivirus biogenesis. J. Virol. 88, 12041-12054. doi: 10.1128/JVI.02061-14

Meoni, G., Ghini, V., Maggi, L., Vignoli, A., Mazzoni, A., Salvati, L., et al. (2021). Metabolomic/lipidomic profiling of COVID-19 and individual response to tocilizumab. PLoS Pathog. 17:e1009243.

Nanda, P., and Ghosh, A. (2021). Genome scale-differential flux analysis reveals deregulation of lung cell metabolism on SARS-CoV-2 infection. PLoS Comput. Biol. 17:e1008860.

Paez-Franco, J. C., Torres-Ruiz, J., Sosa-Hernandez, V. A., Cervantes-Diaz, R., Romero-Ramirez, S., Perez-Fragoso, A., et al. (2021). Metabolomics analysis reveals a modified amino acid metabolism that correlates with altered oxygen homeostasis in COVID-19 patients. Sci. Rep. 11:6350. doi: 10.1038/s41598-02185788-0

Riviere, A., Selak, M., Lantin, D., Leroy, F., and De Vuyst, L. (2016). Bifidobacteria and butyrate-producing colon bacteria: importance and strategies for their stimulation in the human gut. Front. Microbiol. 7:979. doi: 10.3389/fmicb.2016. 00979

Rosas-Salazar, C., Kimura, K. S., Shilts, M. H., Strickland, B. A., Freeman, M. H., Wessinger, B. C., et al. (2021). SARS-CoV-2 infection and viral load are associated with the upper respiratory tract microbiome. J. Allergy Clin. Immunol. 147, 1226.e2-1233.e2.

Schloer, S., Brunotte, L., Mecate-Zambrano, A., Zheng, S., Tang, J., Ludwig, S., et al. (2021). Drug synergy of combinatory treatment with remdesivir and the repurposed drugs fluoxetine and itraconazole effectively impairs SARS-CoV-2 infection in vitro. Br. J. Pharmacol. 178, 2339-2350.

Schloer, S., Goretzko, J., Kühnl, A., Brunotte, L., Ludwig, S., and Rescher, U. (2019). The clinically licensed antifungal drug itraconazole inhibits influenza virus in vitro and in vivo. Emerg. Microbes Infect. 8, 80-93.

Tanner, L. B., Chng, C., Guan, X. L., Lei, Z., Rozen, S. G., and Wenk, M. R. (2014). Lipidomics identifies a requirement for peroxisomal function during influenza virus replication. J. lipid Res. 55, 1357-1365. doi: 10.1194/jlr.M049148

Todorov, H., Kollar, B., Bayer, F., Brandão, I., Mann, A., Mohr, J., et al. (2020). $\alpha$-Linolenic acid-rich diet influences microbiota composition and villus morphology of the mouse small intestine. Nutrients 12:732.

Tummino, T. A., Rezelj, V. V., Fischer, B., Fischer, A., O’Meara, M. J., Monel, B., et al. (2021). Drug-induced phospholipidosis confounds drug repurposing for SARS-CoV-2. Science 373, 541-547.

Villapol, S. (2020). Gastrointestinal symptoms associated with COVID-19: impact on the gut microbiome. Transl. Res. 226, 57-69. doi: 10.1016/j.trsl.2020.08.004

Wang, K., Liao, M., Zhou, N., Bao, L., Ma, K., Zheng, Z., et al. (2019). Parabacteroides distasonis alleviates obesity and metabolic dysfunctions via production of succinate and secondary bile acids. Cell Rep. 26, 222.e5-235.e5.

Wickham, H. (2009). ggplot2. ggplot2. New York, NY: Springer New York, doi: 10.1007/978-0-387-98141-3. 
Winkler, E. S., Shrihari, S., Hykes, B. L. Jr., Handley, S. A., Andhey, P. S., Huang, Y.-J. S., et al. (2020). The Intestinal microbiome restricts alphavirus infection and dissemination through A bile acid-type I Ifn signaling axis. Cell 182, 901.e18-918.e18. doi: 10.1016/j.cell.2020.06.029

Wölfel, R., Corman, V. M., Guggemos, W., Seilmaier, M., Zange, S., Müller, M. A., et al. (2020). Virological assessment of hospitalized patients with COVID-2019. Nature 581, 465-469. doi: 10.1038/s41586-020-2196-x

Xu, K., Cai, H., Shen, Y., Ni, Q., Chen, Y., Hu, S., et al. (2020). [Management of corona virus disease-19 (COVID-19): the Zhejiang experience]. Zhejiang $\mathrm{Da}$ Xue Xue Bao Yi Xue Ban. 49, 147-157. doi: 10.3785/j.issn.1008-9292.2020.02.02

Xu, R., Lu, R., Zhang, T., Wu, Q., Cai, W., Han, X., et al. (2021). Temporal association between human upper respiratory and gut bacterial microbiomes during the course of COVID-19 in adults. Commun. Biol. 4:240. doi: 10.1038/ s42003-021-01796-w

Yan, B., Chu, H., Yang, D., Sze, K. H., Lai, P. M., Yuan, S., et al. (2019). Characterization of the lipidomic profile of human coronavirus-infected cells: implications for lipid metabolism remodeling upon coronavirus replication. Viruses 11:73. doi: 10.3390/v11010073

Yeoh, Y. K., Zuo, T., Lui, G. C., Zhang, F., Liu, Q., Li, A. Y., et al. (2021). Gut microbiota composition reflects disease severity and dysfunctional immune responses in patients with COVID-19. Gut 70, 698-706. doi: 10.1136/gutjnl2020-323020

Zuo, T., Zhang, F., Lui, G. C. Y., Yeoh, Y. K., Li, A. Y. L., Zhan, H., et al. (2020b). Alterations in gut microbiota of patients with COVID-19 during time of hospitalization. Gastroenterology 159, 944.e8-955.e8. doi: 10.1053/j.gastro. 2020.05.048

Zuo, T., Zhan, H., Zhang, F., Liu, Q., Tso, E. Y. K., Lui, G. C. Y., et al. (2020a). Alterations in fecal fungal microbiome of patients with COVID-19 during time of hospitalization until discharge. Gastroenterology 159, 1302.e5-1310.e5.

Conflict of Interest: The authors declare that the research was conducted in the absence of any commercial or financial relationships that could be construed as a potential conflict of interest.

Publisher's Note: All claims expressed in this article are solely those of the authors and do not necessarily represent those of their affiliated organizations, or those of the publisher, the editors and the reviewers. Any product that may be evaluated in this article, or claim that may be made by its manufacturer, is not guaranteed or endorsed by the publisher.

Copyright () 2021 Al Bataineh, Henschel, Mousa, Daou, Waasia, Kannout, Khalili, Kayasseh, Alkhajeh, Uddin, Alkaabi, Tay, Feng, Yousef and Alsafar. This is an openaccess article distributed under the terms of the Creative Commons Attribution License (CC BY). The use, distribution or reproduction in other forums is permitted, provided the original author(s) and the copyright owner(s) are credited and that the original publication in this journal is cited, in accordance with accepted academic practice. No use, distribution or reproduction is permitted which does not comply with these terms. 